\title{
Aneurismas da aorta
}

Januário M. SOUZA*, Salomón O. ROJAS*, Marcos F. BERLINCK*, Ricardo MAZZIERI*, Paulo A. F. OLIVEIRA*, José Renato M. MARTINS*, Dante F. SENRA*, Valéria SALAZAR*, Sérgio Almeida de OLIVEIRA*

SOUZA, J. M.; ROJAS, S. D.; BERLINCK, M. F.; MAZZIERI, R.; OLIVEIRA, P. A. F.; MARTINS, J. R. M.; SENRA, D. F.; SALAZAR, V.; OLIVEIRA, S. A. - Aneurismas da aorta. Rev. Bras. Cir. Cardiovasc., 7 (3):201-207, 1992.

RESUMO: Entre janeiro de 1979 e janeiro de 1992, foram realizadas 212 operaçōes para correção de aneurismas e de dissecçōes da aorta. Neste trabalho serão analisados 104 procedimentos cirúrgicos (em 97 pacientes) para correção de aneurismas. A idade dos pacientes variou de 14 a 79 anos (média 59,5 anos) o sexo predominante foi o masculino, com 75 pacientes. Os aneurismas localizavam-se na aorta ascendente em 46 pacientes, na croça em 8, na aorta descendente em 8, na aorta toráco-abdominal em 8, na aorta abdominal em 21, na aorta descendente e abdominal em 2, na aorta ascendente e tóraco-abdominal em 2, na aorta ascendente e descendente em 1, na aorta ascendente, croça e descendente em 1 . Doenças cardiovasculares associadas estavam presentes em 39 pacientes, sendo valvopatia aórtica em 18 (excluídos os pacientes com ectasia ânulo-aórtica), insuficiência coronária em 17, coarctação da aorta em 2, persistência do canal arterial em 1 e valvopatia mitral e aórtica em 1 . A mortalidade imediata (hospitalar e/ou 30 dias) foi de $14,4 \%$, sendo de $27,7 \%$ (5/18) para pacientes com mais de 70 anos e de $11,3 \%$ (9/79) para pacientes com idade inferior a 70 anos. Os aneurismas localizados na aorta ascendente e croça foram operados como o auxílio de circulação extracorpórea. Parada circulatória e hipotermia profunda foram utilizadas em todos os pacientes com aneurisma da croça. O estudo tomográfico e angiográfico deve ser de toda a aorta, pela possibilidade de aneurismas de localizaçōes múltiplas.

DESCRITORES: aneurismas de aorta, cirurgia.

\section{INTRODUÇĀO}

Rudolph Matas, em 1888, descreveu a abordagem do aneurisma pelo interior de sua cavidade, após controle proximal e distal da artéria, com dissecçāo mínima, evitando, dessa maneira, sangramentos e lesōes de órgāos e nervos aderentes ao aneurisma, tornando a operaçāo um procedimento mais rápido ?. 19. 31. Essa técnica ficou abandonada por vários anos, nāo sendo adotada para a correçāo de aneurismas de aorta, tendo sido introduzida por CREECH Jr. ${ }^{17}$, De BAKEY et alii ${ }^{20}$, COOLEY \& De BAKEY ${ }^{6}$ e CRAWFORD et alii ${ }^{16}$, autores que muito têm contribuído no tratamento dessa doença, nas últimas 4 décadas. $A$ utilizaçāo dos homoenxertos aórticos ${ }^{22,26}$, dos enxertos industrializados ${ }^{8}$, da circulaçāo extracorpórea com hipotermia e parada circulatória, do pinçamento temporário da aorta e medidas para proteçāo do sistema nervoso central ${ }^{15}, 18,29,32,34,35$, possibilitam a correçāo de aneurismas aórticos de qualquer localizaçāo 9, 12,14. 15, 19, 25 inclusive a substituição de toda a aorta ${ }^{13}$.

BICKERSTAFF et $a_{1 i i^{2}}$ mostraram que pacientes portadores de aneurismas da aorta torácica e abdominal nāo tratados apresentam estimativa atuarial de sobrevida aos 5 anos de apenas $19,3 \%$ e $23,9 \%$, respectivamente.

\section{CASUÍSTICA E MÉTODOS}

Entre janeiro de 1979 e janeiro de 1992, foram

Trabalho realizado no Serviço de Cirurgia Cardiovascular do Prof. Dr. Sérgio Almeida de Oliveira. Hospital da Beneficência Portuguesa. São Paulo, SP, Brasil.

Apresentado ao $19^{\circ}$ Congresso Nacional de Cirurgia Cardíaca, São Paulo, SP, 7 a 9 de maio, 1992.

* Do Serviço de Cirurgia Cardiovascular do Prof. Sérgio Almeida de Oliveira.

Endereço para separatas: Januário de Souza. Rua Pará, 49. 01243, Sảo Paulo, SP, Brasil. 
SOUZA, J. M.; ROJAS, S. D.; BERLINCK, M. F.; MAZZIERI, R.; OLIVEIRA, P. A. F.; MARTINS, J. R. M.; SENRA, D. F.; SALAZAR, V.; OLIVEIRA, S. A. - Aneurismas da aorta. Rev. Bras. Cir. Cardiovasc., 7 (3):201-207, 1992.

realizadas 212 operaçōes para correçāo de aneurismas e de dissecçōes da aorta. Neste trabalho serāo analisados 104 procedimentos cirúrgicos (em 97 pacientes) para a correçāo de aneurismas.

Os aneurismas localizavam-se na aorta ascendente em 46 pacientes, na croça em 8 , na aorta descendente em 8, na aorta tóraco-abdominal em 8, na aorta abdominal em 21, na aorta descendente e abdominal em 2, na aorta ascendente e tóraco-abdominal em 2, na aorta ascendente e descendente em 1 e na aorta ascendente, croça e descendente em 1.

O sexo masculino predominou com 75 pacientes. A idade variou de 14 a 79 anos, com média de 59,5 anos, sendo mais elevada nos pacientes comaneurismas torácicos associados a aneurismas abdominais (média, 74,5 anos) e nos pacientes com aneurismas abdominais (média, 66 anos) (Tabela 1).

Doenças cardiovasculares associadas estavam presentes em 39 pacientes, sendo valvopatia aórtica

TABELA 1

ANEURISMAS DA AORTA IDADE EM RELAÇĀO À LOCALIZAÇĀO

\begin{tabular}{lrc}
\hline \multicolumn{1}{c}{ JAN. 79-JAN. 92} & \multicolumn{2}{c}{$N^{\circ}=97$ PACIENTES } \\
LOCALIZAÇĀO & IDADE & MÉDIA IDADE \\
\hline Ao ASC & $14-72 \mathrm{a}$ & 48.5 \\
Croça & $24-75 \mathrm{a}$ & 46.6 \\
Ao DESC & $26-78 \mathrm{a}$ & 61.0 \\
Ao Tóraco-ABD & $31-73 \mathrm{a}$ & 60.1 \\
Ao ABD & $55-79 \mathrm{a}$ & 66.0 \\
Ao DESC + ABD & $71-78 \mathrm{a}$ & 74.5 \\
Ao ASC + Tóraco-ABD & $58-71 \mathrm{a}$ & 64.5 \\
Ao ASC + DESC & $60 \mathrm{a}$ & 60.0 \\
Ao ASC + Croça + DESC & $55 \mathrm{a}$ & 55.0 \\
\hline
\end{tabular}

$A O=$ Aorta $;$ ASC $=$ Ascendente $;$ DESC $=$ Descendente $A B D=$ Abdominal em 18 (excluídos os pacientes com ectasia ânuloaórtica), insuficiência coronária em 17 (sendo que 15 foram revascularizados previamente ou durante a operaçāo para correçāo do aneurisma), coarctaçāo da aorta em 2, persistência do canal arterial em 1 e valvopatia mitral e aórtica em 1.

Dos 46 pacientes com aneurisma da aorta ascendente, 4 eram reoperaçōes e 2 estavam sendo reoperados pela segunda vez; dos 8 pacientes com aneurisma da croça, 3 eram reoperaçōes, dos $8 \mathrm{com}$ aneurisma tóraco-abdominal 2 receberam revascularizaçāo miocárdica prévia e dos 21 com aneurisma da aorta abdominal, 9 tiveram revascularização miocárdica e 2 implante de marcapasso cardíaco.

\section{Etiologia}

Nos 46 pacientes com aneurisma da aorta ascendente, a etiologia foi: ectasia ânulo-aórtica em 18, asterosclerótica ou degenerativa em 27 e sifilítica em 1; nos pacientes com aneurisma da croça da aorta, foi: aterosclerótica em 5 , complicaçāo tardia de correçāo de coarctaçāo em 1 , de correçāo de canal arterial em 1 e de correção de traumatismo da aorta em outro; nos pacientes com aneurisma da aorta descendente, foi: aterosclerótica em 7 e pós-correçăo de coarctação da aorta em 1; nos pacientes com aneurisma tóraco-abdominal, abdominal e mais de um local, a etiologia foi aterosclerótica, exceto em um tóraco-abdominal em que foi sifilítica.

\section{Técnica Operatória}

A técnica operatória variou de acordo com a localizaçāo, doenças associadas e etiologia. Nos pacientes com aneurisma da aorta ascendente, a circulação extracorpórea foi utilizada em todos os pacientes, com hipotermia moderada $\left(25^{\circ} \mathrm{C}\right)$ ou entāo profunda $\left(15^{\circ} \mathrm{C}\right)$ de temperatura esofágica nos casos

TABELA 2

ANEURISMAS DA AORTA ASCENDENTE - PROCEDIMENTO CIRÚRGICO

\begin{tabular}{|c|c|c|c|}
\hline \multicolumn{2}{|l|}{ JAN. 79 - JAN. 92} & \multicolumn{2}{|c|}{$N^{\circ}=46$ PACIENTES } \\
\hline CORREÇĀO CIRÚRGICA & NPACIENTES & ÓBITOS & $\%$ \\
\hline Tubo valvulado + reimp. coronárias & 21 & 2 & $\begin{array}{l}9,5 \\
P=0,50\end{array}$ \\
\hline Tubo+substituiçāo valvar* & 21 & 1 & 4,7 \\
\hline Tubo ** & 4 & 0 & $\cdot$ \\
\hline TOTAL & 46 & $3+$ & 6,5 \\
\hline
\end{tabular}

* Substituição valvar mitral associada - 1 paciente

** Revascularizaçăo miocárdica -2 pacientes

+1 paciente primeira reoperaçảo

+1 paciente segunda reoperação 
em que houve necessidade de parada circulatória; em geral, a canulaçāo foi da artéria femoral e átrio direito ou veia femoral. Foi usada soluçāo cardioplégica (cristalóide nos primeiros anos e nos últimos 4 anos, sangüínea - $\left(\right.$ BUCKBERG $\left.^{3}\right)$. Em 21 pacientes utilizamos tubo valvulado com implante das coronárias; em 21 pacientes, substituição valvar e tubo na aorta acima das coronárias e em 4 pacientes apenas tubo nessa posição; em 2 pacientes foi feita também revascularização do miocárdio e em 1 substituiçăo da valva mitral (Tabela 2).

Todos os pacientes portadores de aneurisma da croça da aorta, foram operados com auxílio da circulaçāo extracorpórea, hipotermia profunda $\left(15^{\circ} \mathrm{C}\right)$ e parada circulatória, sendo que, em 4 os vasos braquiocefálicos foram implantados no tubo. Nesses casos o aneurisma estendia-se para a aorta ascendente, que também foi substituída, e em 2 havia envolvimento da aorta descendente que também foi tratada.

Nos 8 pacientes com aneurisma da aorta descendente, 6 foram operados com pinçamento proximal e distal e 2 com uso de circulação extracorpórea, hipotermia $\left(15^{\circ} \mathrm{C}\right)$ e parada circulatória; 1 desses pacientes apresentava-se com aneurisma roto.

Em 8 pacientes com aneurisma tóraco-abdominal foi utilizado pinçamento aórtico proximal e distal e anastomose do tubo à aorta proximal e distal e reimplante de artérias intercostais e viscerais, de acordo com a extensão do aneurisma. Nós últimos 5 pacientes fizemos monitorização da pressão liquórica, já que eleva-se com o pinçamento proximal da aorta e cai após despinçamento ${ }^{32,35}$.

Nos 21 pacientes portadores de aneurisma da aorta abdominal intra-renal, foi utilizado pinçamento proximal e distal ao aneurisma e tubo, simples ou bifurcado, dependendo do envolvimento das artérias ilíacas e de arteriopatia obstrutiva associada.

Nos pacientes com localizaçōes múltiplas sempre foi operado em primerio tempo o aneurisma proximal e em seguida o distal.

Sempre que possível temos utilizado o tubo de

TABELA 3

ANEURISMAS DA AORTA MORTALIDADE EM RELAÇÃO À IDADE

\begin{tabular}{cccc}
\hline \multicolumn{2}{c}{ JAN. 79- JAN. 92 } & \multicolumn{2}{c}{$N^{\circ}=97$ PACIENTES } \\
IDADE & N PAC. & ÓBITOS & $\%$ \\
\hline$<70$ anos & 79 & 9 & 11.3 \\
& & & $P=0,0841$ \\
$>70$ anos & 18 & 5 & 27.7 \\
TOTAL & 97 & 14 & 14.4 \\
\hline
\end{tabular}

pericárdio corrugado*, pois o sangramento é mínimo, sem necessidade de pré-coagulação e fácil para ser suturado - o incoveniente é que o seu comprimento máximo é de $20 \mathrm{~cm}$, não sendo, portanto, suficiente para correção de aneurismas muito extensos.

\section{Diagnóstico}

O diagnóstico clínico foi sempre confirmado com exames complementares: ultra-som, tomografia computadorizada e cateterismo cardíaco com aortografia, esta, sempre que possível, de toda a aorta, pois existe sempre a possibilidade de aneurismas de localizaçōes múltiplas.

\section{RESULTADOS}

A mortalidade imediata (hospitalar ou 30 dias dias) nos 97 pacientes foi de $14,4 \%$ (14 pacientes), sendo de $27,7 \%$ (5-18) para os pacientes com 70 anos ou mais e de $11,3 \%$ para pacientes com idade inferior a 70 anos (9/79) (Tabela 3). A mortalidade variou também de acordo com a localização (Tabela 4).

$\mathrm{Na}$ correção dos aneurismas da aorta ascendente, em 46 pacientes ocorreram 3 óbitos imediatos, sendo uma reoperação e a causa do óbito infecçāo respiratória: outro paciente que estava sendo operado pela segunda vez faleceu por coagulopatia e o terceiro teve morte súbita no pós-operatório imediato; neste paciente o implante das coronárias foi pela técnica de CABROL et alii ${ }^{5}$. Nesse grupo, $3 / 46$ (6,5\%) pacientes foram reoperados por sangramento pósoperatório, semóbitos.

\section{TABELA 4}

ANEURISMAS DA AORTA MORTALIDADE - LOCALIZAÇĀO

\begin{tabular}{lrcc}
\hline \multicolumn{1}{c}{ JAN. 79-JAN. 92 } & \multicolumn{3}{c}{$N^{\circ}=97$ PACIENTES } \\
IDADE & $N^{\circ}$ PAC. & ÓBITOS & $\%$ \\
\hline Ao ascendente & 46 & 3 & 6,5 \\
Croça & 8 & 2 & 25,0 \\
Ao descendente & 8 & 3 & 37,5 \\
Ao tóraco-abdominal & 8 & 2 & 25,0 \\
Ao abdominal & 21 & 3 & 14,2 \\
Ao descend. + ABD & 2 & 0 & - \\
Ao ASC + tóraco-ABD & 2 & 1 & 50,0 \\
Ao ASC + DESC & 1 & 0 & - \\
Ao ASC + croça + DESC & 1 & 0 & - \\
\hline & & & 14 \\
\hline
\end{tabular}

$A O=A o r t a ; A B D=A b d o m i n a l ; A S C=$ Ascendente; DESC $=$ Descendente.

* Laboratório Labcor 
SOUZA, J. M.; ROJAS, S. D.; BERLINCK, M. F.; MAZZIERI, R.; OLIVEIRA, P. A. F.; MARTINS, J. R. M.; SENRA, D. F.; SALAZAR, V.; OLIVEIRA, S. A. - Aneurismas da aorta. Rev. Bras. Cir. Cardiovasc., 7 (3):201-207, 1992.

Nos 8 pacientes com aneurismas da croça, ocorreram dois óbitos imediatos, sendo um por coma neurológico em conseqüência de parada cardíaca ocorrida no primeiro dia de pós-operatório e o outro por coagulopatia, seguida de falência de múltiplos órgāos; nesse grupo 3 pacientes eram reoperaçōes e nesses não ocorreram óbitos. Dois pacientes necessitaram traqueostomia e assistência ventilatória prolongada, com bom resultado.

Nos pacientes com aneurisma da aorta descendente ocorreram 3 óbitos, 1 em paciente com 74 anos e em 2 com 78 anos de idade; um dos pacientes tinha aneurisma roto e morreu por insuficiência respiratória, outro morreu também por insuficiência respiratória após 2 meses de assistência ventilatória e o terceiro paciente morreu por embolia pulmonar no $8^{\circ}$ dia de pós-operatório. Os 2 pacientes que faleceram por insuficiência respiratória foram operados com auxílio de circulação extracorpórea, hipotermia profunda $\left(15^{\circ} \mathrm{C}\right)$ e parada circulatória. Nos 6 pacientes operados apenas com pinçamento aórtico ocorreu um óbito (embolia pulmonar), sendo essa a técnica preferida quando possível 6, 14, 27.

Em 8 pacientes com aneurisma da aorta tóraco- abdominal ocorrem 2 óbitos, sendo um por insuficiência respiratória em paciente já submetido a revascularizaçāo miocárdica e que tinha também paquipleurite associada e o outro com 73 anos de idade por infecçāo respiratória, na terceira semana de pósoperatório.

Nos 21 pacientes submetidos a correção de aneurisma da aorta abdominal ( 9 com revascularizaçāo miocárdica prévia e 2 com implante de marcapasso cardíaco) ocorreram 3 óbitos, 1 por infecçāo respiratória em paciente de 79 anos de idade e 2 por insuficiência renal aguda, sendo que 1 desses pacientes era portador de insuficiência renal crônica compensada. Um paciente apresentou obstruçāo intestinal por bridas no pós-operatório, sendo operado e teve boa evoluçāo.

Em 2 pacientes com aneurismas da aorta descendente e abdominal, operados em dois tempos, não houve óbitos.

Em 2 pacientes operados inicialmente para correçāo de aneurisma da aorta ascendente e dois e três meses após correção de aneurisma tóraco-abdominal, ocorreu um óbito em paciente em que houve

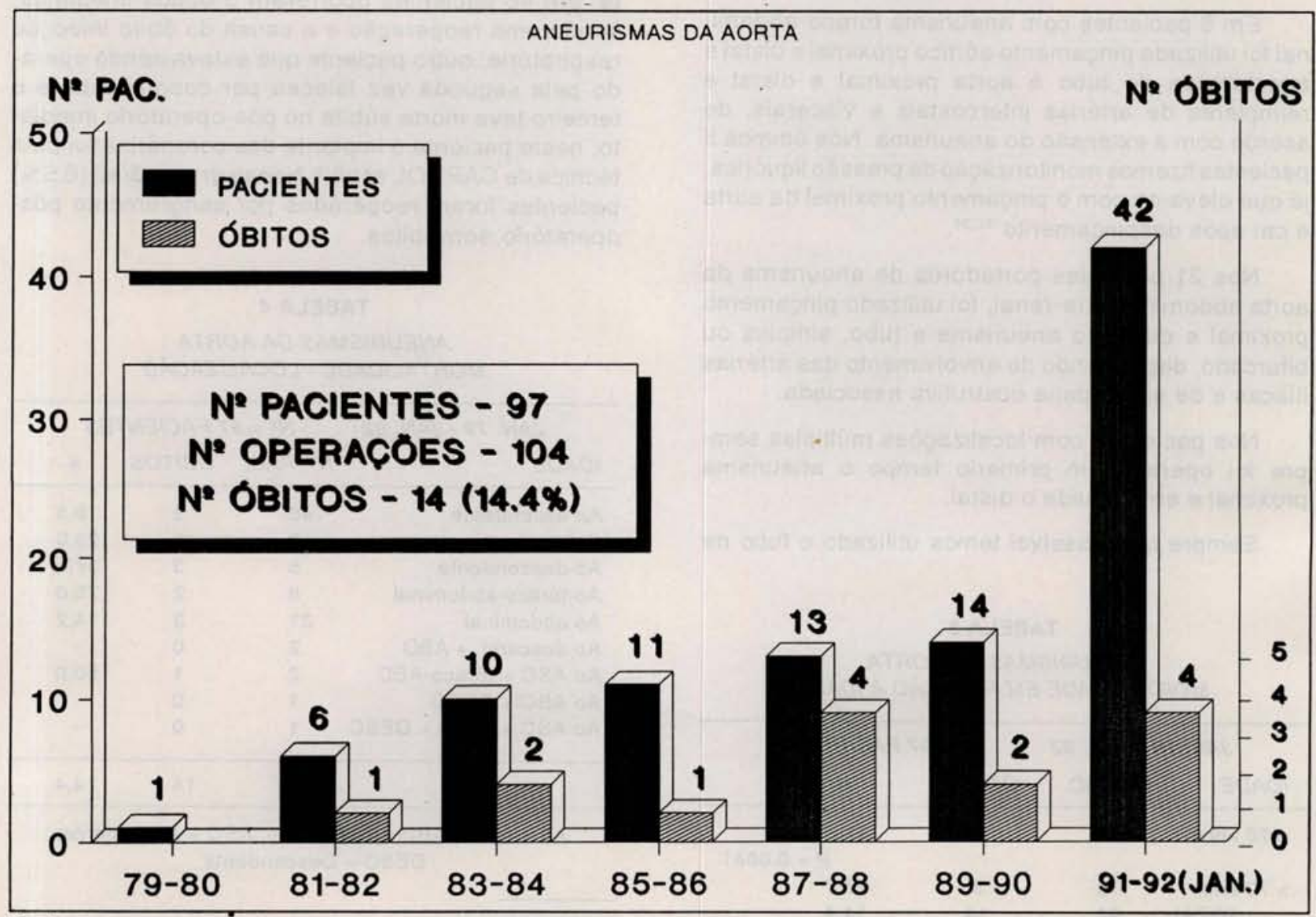

Fig. 1 
necessidade do uso de circulação extracorpórea e hipotermia com parada circulatória; esse paciente faleceu em coma neurológico, seqüela de parada cardíaca por hipóxia causada por hemorragia intrapulmonar durante o fechamento da incisão tóracoabdominal.

Um paciente foi operado inicialmente para correção de aneurisma da aorta ascendente e seis anos após, correção de aneurisma da aorta descendente; em ambas as operaçōes foi usada circulaçāo extracorpórea sendo que na segunda foi utilizada hipotermia profunda com parada circulatória. Em outro paciente, inicialmente corrigiu-se aneurisma da croça e da aorta ascendente, com substituiçāo da valva aórtica, usando-se circulação extracorpórea com hipotermia profunda e parada circulatória e três meses depois foi feita correção do aneurisma da aorta descendente com pinçamento aórtico proximal e distal. Esses dois últimos pacientes não tiveram qualquer complicação.

Como já foi relatado, 3 pacientes com aneurisma da aorta ascendente necessitaram reoperação por sangramento; nos demais apenas 1 com correçāo de aneurisma tóraco-abdominal necessitou reoperação por sangramento de artéria da parede torácica (artéria intercostal).

\section{COMENTÁRIOS}

Em nosso Serviço, no último ano, aumentou muito o número de pacientes portadores de aneurismas da aorta; dos 97 casos aqui relatados, 42 foram operados entre janeiro de 1991 e janeiro de 1992, quando ocorreram $4(9,5 \%)$ óbitos (Figura 1$)$.

Desde que foi descrita em 1968, por BENTAL \& De BONO ', a substituição da aorta ascendente por tubo valvulado e reimplante das artérias coronárias tem sido a melhor técnica para correção da ectasia ânulo-aórtica ${ }^{24} \mathrm{e}$ aneurismas que envolvem os seios de Valsalva e a valva aórtica ${ }^{30}$, pois todo tecido doente é ressecado evitando-se, dessa maneira, a reoperaçāo tardia.

A técnica da inclusão do tubo tem sido criticada pela possibilidade de formaçāo de pseudoaneurismas tardios ${ }^{28}$. A manobra de CABROL et alii ${ }^{4}$, fazendo uma fístula entre o espaço peritubo e o átrio direito, reduz a incidência de hemorragia e pode evitar a formação de pseudoaneurismas ${ }^{8,12,13,18,30}$. A modificação proposta por $\mathrm{CABROL}$ et alii ${ }^{5} \mathrm{em}$ que um tubo de Dacron interposto entre os óstios coronarianos é anastomosado ao tubo que substitui a aorta, pode ser útil em casos especiais, mas não o temos utilizado de rotina.

A correção dos aneurismas da croça teve um grande avanço desde que GRIEP et alii ${ }^{25}$ propuseram a utilização da circulação extracorpórea com hipotermia e parada circulatória, tornando essa operação mais simples e com baixa mortalidade ${ }^{12}$. 15; a nossa experiência é de 8 casos, sendo que os óbitos foram, um por coma em conseqüência de parada cardíaca no pós-operatória e outro por coagulopatia.

Os aneurismas tóraco-abdominais, apresentam a maior dificuldade para correção, principalmente pela possibilidade de complicaçōes hemorrágicas, pulmonares e neurológicas ${ }^{11}, 19,23,33,35$. CRAWFORD et alii " têm a maior e melhor experiência na correção desses aneurismas e temos procurado seguir os seus ensinamentos. Na nossa experiência inicial, operamos 8 pacientes com dois óbitos, sendo um por insuficiência respiratória em paciente que tinha paquipleurite associada e o outro por infecção respiratória. Em outros 2 pacientes que tinham sido operados previamente por aneurismas da aorta ascendente, ocorreu um óbito durante a correção do aneurisma tóraco-abdominal, por coma neurológico após parada cardíaca no transoperatório.

Nos aneurismas da aorta abdominal, 2 pacientes faleceram por insuficiência renal e 1 por infecçāo respiratória. $A$ insuficiência renal é uma complicação que ocorre com certa freqüência nesse tipo de operação e é de mau prognóstico ${ }^{21}$.

Em conclusāo, podemos dizer que a reoperação, a idade avançada, doenças associadas, infecção respiratória e insuficiência renal aumentam a mortalidade dos pacientes submetidos a correção dos aneurismas da aorta. 
SOUZA, J. M.; ROJAS, S. D.; BERLINCK, M. F.; MAZZIERI, R.; OLIVEIRA, P. A. F.; MARTINS, J. R. M.; SENRA, D. F.; SALAZAR, V.; OLIVEIRA, S. A. - Aneurismas da aorta. Rev. Bras. Cir. Cardiovasc., 7 (3):201-207, 1992.

\section{RBCCV/44205-180}

SOUZA, J. M.; ROJAS, S. D.; BERLINCK, M. F.; MAZZIERI, R.; OLIVEIRA, P. A. F.; MARTINS, J. R. M.; SENRA, D. F.; SALAZAR, V.; OLIVEIRA,, S. A. - Aortic aneurysms. Rev. Bras. Cir. Cardiovasc., 7(3): 201-207, 1992.

ABSTRACT: Among 212 patients undergoing operation for aortic aneurysm and aortic dissection between January 1979 and January 1992, 97 were operated on for aneurysms. The aneurysms were localized in: ascending aorta in $\mathbf{4 6}$ patients, transverse aortic arch in $\mathbf{8}$, descending aorta in 8 , thoracoabdominal aorta in 8 , abdominal (infrarenal) aorta in 21, descending and abdominal aorta in 2, ascending and thoracoabdominal aorta in 2, ascending and descending in 1, ascending, transverse arch and descending aorta in 1. Hospital mortality was $14,4 \%$ being $27,7 \%(5 / 18)$ among patients over 70 years old an $11,3 \%(9 / 79)$ among patients under 70 years of age. Our experience suggests that: secondary and tertiary operations, advanced age, associated diseases, respiratory infection and acute renal failure increase early mortality.

DESCRIPTORS: aneurysms, aortic, surgery.

\section{REFERÊNCIAS BIBLIOGRÁFICAS}

1 BENTALL, H. \& De BONO, A. - A technique for complete replacement of the ascending aorta. Thorax, 23: $338-339,1968$.

BICKERSTAFF, L. K.; PAIROLERO, P. C.; HOLLIER, L. H.; MILTON, L. S.; VAN PEENEN, H. S.; CHERRY, K. S.; JOYCE, S. W.; LIE, I. T. - Thoracic aortic aneurysms: a population - based study. Surgery, 92: 1103-1108, 1982.

BUCKBERG, G. D. - Strategies and logic of cardioplegic delivery to prevent, avoid and reverse ischemic and reperfusion damage. J. Thorac. Cardiovasc. Surg., 93: 127-139, 1987.

CABROL, C.; GANDSBAKHC, I.; PAVIE, A. - Surgical treatment of ascending aortic pathology. J. Cardiovasc. Surg., 3: 168-180, 1988.

CABROL, C.; PAVIE, A.; GANDJBAKCH, I. - Complete replacement of the ascending aorta with reimplantation of the coornary arteries. J. Thorac. Cardiovasc. Surg., 81: 309-315, 1981.

COOLEY, D.A . \& De BAKEY, M. E. - Surgical considerations of intrathoracic aneurysms. Am. Surg., 135: $660-680,1952$

COOLEY, D. A. \& De BAKEY, M. E.; CHEECH Jr., O. Surgical treatment of aortic aneurysms. Surg., 22: 1043-1051, 1956.

COOLEY, D. A.; ROMAGNOLI, A.; MILAM, J. D.; BOSSART, M. S. - A method of preparing woven Dacron aortic grafts of prevent interstitial hemorrhage. Cardiovasc. Dis. Bul. Texas. Heart Inst., 8: $48-52,1981$. graft replacement of aortic root using reparate Dacron

tube for coronary artery reattachment. Ann. Thorac. Surg., 47: 558-565, 1989.

10

CRAWFORD, E. S.; COSELLIM, J. S.; SAFI, H. J. Partial cardioplumonary bypass, hypothermic circulatory arrest and postero-lateral exposure for thoracic aortic aneurysm operation. J. THorac. Cardiovasc. Surg., 94: 824-827, 1987.

CRAWFORD, E. S.; CRAWFORD, J. L.; SAFI, H. J. Thoracoabdominal aortic aneurysms: preoperative and intraoperative factors determining intermediate and long-term results in 605 patients. J.Vasc. Surg., 3: $389-404,1986$.

CRAWFORD, E. S.; CRAWFORD, J. L.; SAFI, H. J.; COSELLI, J. S. - Redo operations for recurrent aneurysmal disease of the ascending aorta and transverse aortic arch. Ann. Thorac. Surg., 40: 439-455, 1985.

13 CRAWFORD, E. S.; CRAWFORD, J. L.; STOWE, C. L.; SAFI, H. S. - Total aortic replacement for chronic aortic dissection occurring in patients with and without Marfan's syndrome. Ann. Surg., 199: 358-362, 1984.

14 CRAWFORD, E. S. \& RUBIO, P. A. - Reappraisal of adjuncts to avoid ischemic in the treatment of aneurysms of descending thoracic aorta. J. Thorac. Cardiovasc. Surg., 66: 693-698, 1983.

15 CRAWFORD, E. S.; STOWE, C. L.; CRAWFORD, J. L.; TITUS, J.; WEILBAECHER, D. G. - Aortic arch aneurysms. Ann. Surg., 199: 742-752, 1984.

16 CRAWFORD, E. S.; SVENSON, L. G.; COSELLI, J. S.; SAFI, H. J.; HESS, K. R. - Surgical treatment of aneurysms and or dissection of the ascending aorta transverse aortic arch and ascending aorta anel transverse aortic arch. J. Thorac. Cardiovasc. Surg., 98: $659-674,1989$. 
SOUZA, J. M.; ROJAS, S. D.; BERLINCK, M. F.; MAZZIERI, R.; OLIVEIRA, P. A. F.; MARTINS, J. R. M.; SENRA, D. F.; SALAZAR, V.; OLIVEIRA, S. A. - Aneurismas da aorta. Rev. Bras. Cir. Cardiovasc., 7 (3):201-207, 1992.

CHEECH Jr, O. - Endoaneurysmorrhaphy and treatment of aortic aneurysms. Ann. Surg., 164: 935-946, 1966.

CUNNINGHAM, J. N.; LASCHINGER, J. C.; MERKIN, H. A.; NATHAN, I. M.; CALUIN, S.; RANSOHUFF, J.; SPENCER, F. C. - Measurement of spinal cord ischemia during operations upon the thoracic aorta. Ann. Surg., 196: 285-296, 1982.

DE BAKEY, M. E.; CRAWFORD, E. S.; GARRET, H. E.; BEALL, A. C.; HOWELL, J. E. - Surgical considerations in the treatment of aneurysms of the thoracoabdominal aorta. Ann. Surg., 162: 650-662, 1965.

DE BAKEY, M. E.; McCOLLUM, C. H.; GRAHAM, J. M. - Surgical treatment of aneurysms of the descending thoracic aorta: long term results in 500 patients. $J$. Cardiovasc. Surg., 19: 571-576, 1978.

DIEHL, J. T.; CALI, R. F.; HERTZER, N. R.; BEVEN, E. G. - Complications of abdominal aortic reconstruction. Ann. Surg., 197: 49-56, 1983.

DUBOST, C.; ALLARY, M.; DECONOMOS, N. Y. - Resection of an aneurysm of the abdominal aorta: restablishment of the continuity by a preserved human arterial graft, with result after five months. Arch. Surg., 64: 405-408, 1952.

ETHEREDGE, S. N.; YEE, S.; SMITH, J. V.; SCHONBERGER, S.; GOLDMAN, M. S. - Sucessful resection of a large aneurysm of the upper abdominal aorta and replacement with homograft. Surgery, 38: 1071$1081,1955$.

GOTT, V. L.; PYERITZ, R. E.; CAMERON, D. E.; GREENE, P. S.; MCKUSICK, V. A. - Composite graft repair of Marfan aneurysm of the ascending aorta: results in 100 patients. Ann. Thorac. Surg., 52: 38$45,1951$.

GRIEP, R. B.; STINSON, E. B.; HOLLINGSWORTH, J. F.; BUEHLER, D. - Prosthetic replacement of the aortic arch. J. Thorac. Cardiovasc. Surg., 70: 1051$1063,1075$.

GROSS, R. E.; BILL, A. H., Jr.; PEIRCE, E. C. - Method of preservation and transplantation of arterial grafts: observations on arterial grafts in dogs: report of transplantation of preserved arterial grafts in nine human cases. Surg. Gynecol. Obstet., 88: 689-704, 1949.
JUCÁ, E. R.; CARVALHO Jr., W.; LORO F', G.; TORRES, J. M.; MEMORIA, S. M.; SIMẢO, F.; OLIVEIRA, F. M. - Ressecção de aneurismas da aorta torácica descendentes pela técnica modificada de Crawford. Arq. Bras. Cardiol., 49: 29-31, 1983.

28

KOUCHOUKOS, N. T.; MARSHALL, W. G.; WEDIGESTECHER, T. A. - Eleven-year experience with composite graft replacement of the ascending aorta and aortic valve. J. Thorac. Cardiovasc. Surg., 92: 691-705, 1986.

KOUCHOUKOS, N. T.; WAREING, T. H.; IZUMOTO, H KLAUSING, W.; ABBOUD, N. - Elective hypothermic cardiopulmonary bypass and circulatory arrest for spinal cord protection during operation on the thoracoabdominal aorta. J. Thorac. Cardiovasc. Surg., 99: 659-664, 1990.

MATAS, R. - Traumatic aneurysm of the left brachial artery. Med. News, 53: 462-465, 1888.

McCULLOUGH, J. L.; HOLLIER, L. H.; NUGENT, M. Paraplegia after thoracic aortic occlusion: influence of cerebrospinal fluid drainage. J. Vasc. Surg., 7: 153-160, 1988.

LEWIS, C. T. P.; COOLEY, D. A.; MURPHY, M. C.; TOLEDO, O.; VEGA, D. - Surgical repair of aortic root aneurysm in 280 patients. Ann.Thorac. Surg., 53: $38-46,1992$.

SVENSON, L. G.; RICHARDS, E.; COULL, A.; ROGERS, G.; FIMMEL, E. L.; HONDER, R. - Relationship of spinal cord blood flow to vascular anatomy during thoracic cross-clamping and shunting. J. Thorac. Cardiovasc. Surg., 91: 71-78, 1986.

USUI, A.; HOTTA, T.; HIROURA, M.; MURASE, M.; MAEDA. M.; KOYAMA, T.; TANAKA, M.; TAKENCHI, E.; YASUURA, K.; WATANABE, T.; ABE, T. - Retrograde cerebral perfusion through a superior vena caval cannula protects the brain. Ann. Thorac. Surg., 53: 47-53, 1992.

35 WOLOSZYN, T. T.; MARINI, C. P.; COONS, M. S.; NATHAN, I. M.; BASU, I. M.; ACINAPURA, A. S.; CUNNINGHAM, J. N. - Cerebrospinal fluid drainage and steroids provide better spinal cord protection during aortic cross-clamping than does either treatment alone. Ann. Thorac. Surg., 49: 78-83, 1990. 